\title{
The experience of training specialists in bioinformatics in Kazakhstan
}

\author{
A. Ivashchenko*, R. Niyazova, Sh. Atambayeva, A. Pyrkova \\ al-Farabi Kazakh National University, Almaty, Kazakhstan \\ *e-mail:a_ivashchenko@mail.ru
}

Key words: bioinformatics, training, courses

Specialists are trained in bioinformatics in al-Farabi Kazakh National University (alFarabi KazNU) for more than 15 years. al-Farabi KazNU has experience in teaching bioinformatics and develops scientific projects in biology, biotechnology and other fields of life sciences using bioinformatics methods. Bioinformatics courses are taught for bachelors, masters and PhD students. Bachelors, masters and PhD students trained by the educational program "Bioinformatics". The following courses are taught: "Fundamentals of Programming", "International Centers for Biotechnology Information", "Computational Technologies in Genomics", "Computational Technologies in Proteomics", "Bioinformatics in Life Sciences", "Bioinformatics in Biology", "Bioinformatics in Biotechnology", "Bioinformatics in Medicine", "Bioinformatics in Agriculture", "Prospects for Development of Bioinformatics", "Signal Systems in Cells and the Body". The department of bioinformatics studies the problems of exonintron organization of genes, establishes the characteristics of the interaction of miRNAs with mRNAs genes. It is elucidated the interaction of miRNAs with mRNAs genes involved in the development of various pathologies: oncological diseases (lung cancer, gastrointestinal cancer, breast cancer, prostate cancer, etc.), cardiovascular diseases (myocardial infarction, atherosclerosis, ischemic heart disease, arterial hypertension, stroke), neurodegenerative diseases (Alzheimer's disease, Parkinson's disease, multiple sclerosis, neurodegenerative diseases caused by repeats of trinucleotides, etc.). The conservation of miRNAs and their interaction with mRNAs genes is studied as an indicator of the evolution of the gene expression regulation system through miRNA, taking into account the dual origin of miRNA (intergenic and intragenic miRNAs). One of the goals is to elucidate the role of multiple miRNA binding sites in mRNAs, to demonstrate the involvement of the entire miRNA nucleotide sequence in binding with mRNAs genes. 